\title{
Lower risk of primary Sjogren's syndrome in patients with dengue virus infection: a nationwide cohort study in Taiwan
}

\author{
Chi-Ching Chang ${ }^{1,2}$ (D) $\cdot$ Yu-Chun Yen ${ }^{3} \cdot$ Cheng-Yi Lee ${ }^{4,5} \cdot$ Chiou-Feng Lin ${ }^{6,7} \cdot$ Chao-Ching Huang ${ }^{8} \cdot$ Ching Wen Tsai $^{3}$. \\ Ting-Wu Chuang ${ }^{9} \cdot$ Chyi-Huey Bai ${ }^{10}$
}

Received: 9 April 2020 / Revised: 29 June 2020 / Accepted: 6 July 2020 / Published online: 15 July 2020

(C) The Author(s) 2020

\begin{abstract}
The data concerning the association between dengue viruses (DV) infection and autoimmune diseases (ADs) remain unclear and are scarce. This nationwide population-based cohort study assessed the risk of ADs among patients with DV infection. We analyzed Taiwanese medical data from the Registry of the National Notifiable Disease Reporting System of Taiwan's Centers for Disease Control between 1998 and 2015 and identified patients with DV infection. From the entire general population data in the National Health Insurance Research Database, we randomly selected a comparison cohort that was individual matching by age, sex, residence, and index date. We analyzed the risk of ADs using a Cox proportional hazards regression model stratified by sex, age, and residence. We enrolled 29,365 patients with DV infection (50.68\% men; mean age, 44.13 years) and 117,460 age-, sex-, and residence-matched controls in the present study. The incidence rates of organ-specific ADs were nonsignificantly higher in the DV cohort than in the non-DV control cohort. An approximately $70 \%$ lower risk of primary Sjogren syndrome (pSS) was evident in the DV cohort than in the non-DV control cohort with an adjusted hazard ratio of 0.30 (95\% confidence interval $0.13-$ 0.67) after adjusting for comorbidities in matched design. By contrast, the other systemic ADs were nonsignificantly lower in the DV cohort than in the non-DV control cohort. This nationwide long-term cohort study demonstrated that patients with DV infection had a lower risk of primary Sjogren syndrome than those without DV infection.
\end{abstract}

\section{Key Points}

- This retrospective, longitudinal cohort observational study shows that patients with DV infection had a lower risk of pSS than those without DV infection.

- The DV cohort had an approximately $70 \%$ lower risk of pSS than the control group, with a multivariate-adjusted HR of 0.30 .

- On the basis of this result, we contended that DV infection has a protective effect that reduces the risk of pSS.

Keywords Autoimmune disease $\cdot$ Cohort study $\cdot$ Dengue virus $\cdot$ Risk

\section{Introduction}

Dengue viruses (DVs), a group of four serologically distinct but related flaviviruses, are responsible for a major emerging viral disease [1]. This mosquito-borne disease has a

Ting-Wu Chuang and Chyi-Huey Bai contributed equally to this work.

Electronic supplementary material The online version of this article (https://doi.org/10.1007/s10067-020-05282-2) contains supplementary material, which is available to authorized users.

Chyi-Huey Bai

baich@tmu.edu.tw

Extended author information available on the last page of the article considerable negative effect on populations in tropical and subtropical areas of the world in terms of illness, mortality, and economic costs, mainly because of the lack of an approved vaccine or antiviral drugs [2]. Infection with one of the four serotypes of DVs results in symptoms ranging from an acute self-limiting febrile illness, dengue fever, to severe dengue hemorrhagic fever or dengue shock syndrome [3, 4].

When a DV infects a host, it may induce the production of autoantibodies because of the structural antigen similarity between viral proteins and self-antigens [5]. The molecular mimicry between the DV protein and self-antigens can cause antibody cross-reactions that lead to platelet dysfunction, endothelial cell apoptosis, defective coagulation, and macrophage activation $[6,7]$. The sequential infection with different DV serotypes may alter the cytokine response of cross-reactive CD4+ T 
cells, resulting in the production of pro-inflammatory cytokines such interleukin (IL)-2, IL-4, IL-6, IL-8, IL-10, IL-13, IL-18, monocyte chemoattractant protein-1, macrophage migration inhibitory factor, transforming growth factor- $\beta$, TNF- $\alpha$, and IFN- $\gamma[8]$.

Some evidence suggests that DVs play key roles in the pathogenesis of autoimmune diseases (ADs), and various mechanisms have been invoked to explain these observations, including molecular mimicry and an increase in the immunogenicity of autoantigens caused by inflammation in the target organ [6]. Paradoxically, infectious agents may also play both a causative and protective role in the pathogenesis of ADs [8]. Numerous epidemiologic and experimental studies have clarified and extended hygiene hypothesis support the microorganism had a diverse immunoregulatory effect on ADs [8, 9].

Because of the high prevalence of DV infections in Taiwan, we have debated whether DV infection is associated with the development of ADs. In Taiwan, patients with dengue are placed under the surveillance of the Centers for Disease Control, R.O.C. (Taiwan; known as the Taiwan CDC) which entails being subject to a routine laboratory-based screening and diagnosis system [10]. All hospital-diagnosed cases of dengue must be reported to the Taiwan CDC for confirmation and subsequent surveillance. With respect to whether DVs are important in the pathogenesis of ADs, we used a nationwide population-based dataset of insurance claims to investigate the association of ADs among patients with DV infection.

\section{Methods}

\section{Data sources}

Medical claims data were obtained from the National Health Research Institutes (NHRI). After receiving approval for this study from the NHRI, we used scrambled patient identification numbers to assess the data, including inpatient care claims and the Registry for Beneficiaries. The NHRI maintains and updates the National Health Insurance (NHI) Research Database (NHIRD). The insurance program maintains contracts with $97 \%$ of the hospitals and clinics in Taiwan [11]. The accuracy and high validity of diagnoses in the NHIRD have been evaluated $[12,13]$. The International Classification of Diseases, Ninth Revision, Clinical Modification (ICD-9CM) codes were used as the diagnosis codes in the present study. The claims data for all 23,740,000 insured persons were used to establish the study cohorts. The research protocol was approved by the Taipei Medical University-Joint Institutional Review Board (N201602014) and was performed in accordance with the approved guidelines. The need for written informed consent was waived by the Institutional Review Board that approved this study's protocols. Informed consent from the study patients was not required because the dataset consisted of deidentified secondary data released for research purposes.

\section{Study cohort}

\section{Dengue case}

Dengue fever is a category 2 notifiable infectious disease in Taiwan and must be reported to the Taiwan CDC by physicians within $24 \mathrm{~h}$ of identifying a suspected case. Suspected dengue cases are confirmed by the Taiwan CDC on the basis of the presence of anti-dengue IgM, nucleotide sequence, or viral isolation. Since 2014, nonstructural protein 1 antigen detection has been used as a rapid diagnostic technique in Taiwan. The patient's travel history is also obtained to clarify whether the case was imported or locally acquired. Only indigenous cases retrieved from the National Notifiable Disease Reporting System of the CDC between 1998 and 2015 were included in the study. The historical medical records (both inpatient and outpatient) of the dengue-confirmed cases can be accessed from the NHIRD. The index date for the DV cohort was identified as the date on which a patient received the first diagnosis of DV infection, as documented in the Registry of the National Notifiable Disease Reporting System. Patients who had been diagnosed with ADs before or within 1 year of DV diagnosis were excluded from this study. The data consolidation and control group selection were processed anonymously in the Department of Statistics, Ministry of Health and Welfare.

\section{Comparison group}

For each patient with a diagnosis of DV infection, a maximum of four comparisons was randomly selected from the NHIRD, a pool of approximately 23.7 million individuals. For the comparison cohort, the index date corresponded to the date on which controls utilized the NHI services. The comparison cohort was age ( \pm 2 years), sex (exact), residence (exact), and index date-matched to the DV cohort. Patients diagnosed with an AD before were excluded. The objective of this matching process by propensity score was to guarantee a similar baseline follow-up duration.

\section{Study endpoints}

Each study patient was followed until one of the following outcomes occurred: an AD was diagnosed, the patient was lost to follow-up, the patient died, and the patient withdrew from the NHI system. We identified patients with ADs using ICD9-CM codes. ADs in this study were categorized into two broad types: systemic and organ-specific ADs [14]. The systemic ADs included Sjogren syndrome (SS; ICD-9-CM code 710.2), psoriasis (ICD-9-CM codes 694.3, 696.0, and 696.1), 
rheumatoid arthritis (RA; ICD-9-CM code 714.0), systemic lupus erythematosus (SLE; ICD-9-CM code 710.0), scleroderma (ICD-9-CM code 710.1), and polymyositis (PM; ICD-9-CM code 710.4). In Taiwan, patients with systemic ADs (except ankylosing spondylitis ICD-9-CM code 720.0, $720.2,720.8,720.9$, and psoriasis ICD-9-CM codes 696.0, 696.1, and 694.3) are eligible for a catastrophic illness certificate after receiving the diagnosis from a rheumatology specialist based on their clinical manifestations, laboratory data, and international criteria; the certification requires the precise fulfillment of the related classification criteria [15-24]. The organ-specific ADs included Addison's disease (ICD-9-CM code 255.4), autoimmune hemolytic anemia (ICD-9-CM code 283.0), diabetes mellitus type 1 (DM; ICD-9-CM code 250.0, 357.2 , 366.41, 583.81), Graves' disease (ICD-9-CM code 242.0), Hashimoto's thyroiditis (ICD-9-CM code 245.2, 780.01, 244.8, 244.9), Henoch-Schonlein purpura (ICD-9CM code 287.0), immune thrombocytopenic purpura (ICD9-CM code 287.3), autoimmune hepatitis (ICD-9-CM code 571.49), myasthenia gravis (ICD-9-CM code 358.0), and inflammatory bowel disease (IBD; ICD-9-CM codes 555 and 556). A person was considered to have a new onset of an organic $\mathrm{AD}$ only if the condition occurred in an inpatient setting or was noted in three or more outpatient visits.

In addition, patients with the comorbidities of SLE, RA, scleroderma, PM, DM, a history of head and neck radiation treatment, hepatitis $\mathrm{C}$ infection, AIDS, pre-existing lymphoma, sarcoidosis, graft versus host disease, and anticholinergic drug use were excluded to limit our study sample to primary SS (pSS). Therefore, the catastrophic illness patient data are highly accurate and reliable $[25,26]$.

\section{Statistical analysis}

We compared the frequency or mean of demographic status (age, comorbidity, residence, sex, and periodontitis) between the DV and non-DV cohorts using the chi-squared test or $t$ test. The standardized mean differences (SMD) in a matched sample were also shown. The incidence rates of organ-specific and systemic ADs were estimated during the follow-up duration in the DV and non-DV cohorts. The stratified Cox proportional hazards regression model was used to estimate the corresponding hazard ratios (HRs) and 95\% confidence intervals (CIs). The matched age, gender, and residence are stratified, and CCI score and periodontitis are adjusted in the Cox regression model. Besides, for the type 1 error problem of multioutcomes especially under the category of systemic autoimmune diseases, the Hochberg corrected $p$ was calculated.

Finally, the cumulative incidences of organ-specific and systemic ADs were estimated using the Kaplan-Meier estimator, known as the product limit estimator, in the DV and nonDV cohorts. SAS (version 9.4, SAS Institute, Cary, NC, USA) was used for all the data analyses, and $p<0.05$ was considered statistically significant.

\section{Results}

\section{Baseline characteristics of patients with DV infection and comparison cohorts}

A total of 29,365 patients with DV infection were selected from the Registry of Disease Reporting System of the Taiwan CDC and 117,460 control subjects were selected from the NHIRD. The mean follow-up duration in patients with DV infection and without DV infection cohorts was $4.52 \pm 4.36$ and $4.56 \pm 4.32$ years, respectively.

The flowchart of the study population selection is presented in Fig. 1. The demographic characteristics and baseline comorbidities of the study cohorts are listed in Table 1 . The mean ages in the DV and control cohorts were $44.12 \pm 19.05$ and $44.13 \pm 19.05$ years, respectively, and the sex ratio of the study population was similar (male $50.68 \%$ and female $49.32 \%$ ). All standardized mean difference in age, gender, and the resident was lower than 0.001 . CCI score and periodontitis baseline characteristics were significantly different.

\section{Incidence rates and adjusted HRs of ADs in the DV and non-DV cohorts}

During the study period, the incidence rate of overall systemic ADs was significantly lower in the DV cohort than in the nonDV cohort (12.60 vs 15.03) with an adjusted HR of 0.81 (95\% CI 0.69-0.96) after matching age, gender, residence, and adjusting for comorbidities (Table 2). Furthermore, for various systemic ADs, $n=80$ and $n=7$ patients in the non-DV and DV cohorts, respectively, developed pSS. The incidence rate of pSS was lower in the DV cohort than in the non-DV cohort (0.51 vs 1.47), with an adjusted HR of 0.30 (95\% CI 0.13 0.67 ) after matching age, gender, residence, and adjusting for comorbidities. It demonstrated DV patients had $70 \%$ less likely to have pSS than control groups significantly (Hochberg $p<0.001)$. By contrast, the other systemic ADs were nonsignificantly lower in the DV cohort than in the non-DV control cohort (Table 2).

For organ-specific ADs, the incidence rate of overall organspecific ADs was nonsignificantly higher in the DV cohort than in the non-DV control cohort (Table 2). Furthermore, the incidence rate of respective organ-specific ADs was also nonsignificantly higher in the DV cohort than in the non-DV control cohort. Detailed information such as model parameters and related standard error, Walt statistics and $p$ value were appended in the supplementary table. 
Fig. 1 Study design

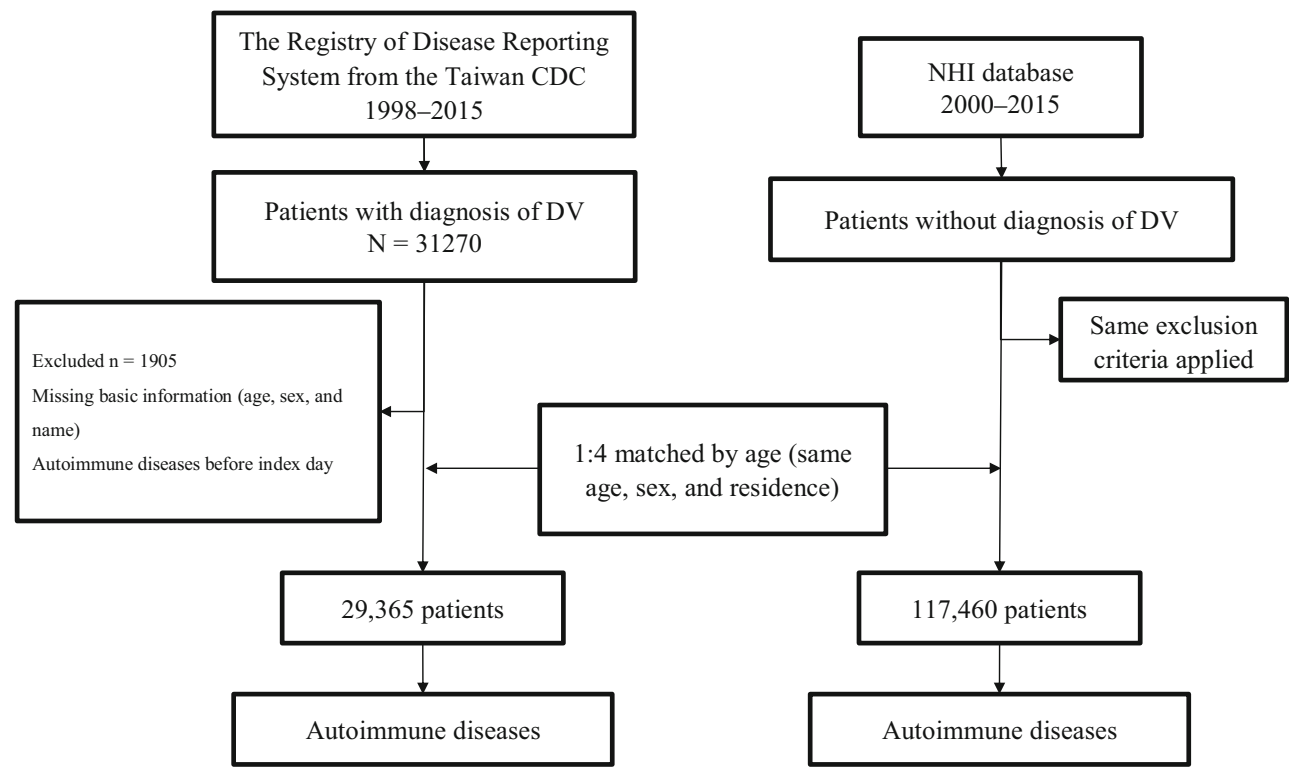

\section{Cumulative incidences of ADs in the DV and non-DV cohorts}

The comparative cumulative incidence of organ-specific ADs in the DV and non-DV cohorts is presented in Fig. 2. The Kaplan-Meier estimates of overall and respective organ-specific AD-free survival revealed a nonsignificantly higher incidence rate in the DV cohort than in the matched control cohort (Fig. 2a-h). The comparative cumulative incidence of systemic ADs in the DV and nonDV cohorts is presented in Fig. 2. The Kaplan-Meier estimates of overall systemic AD-free survival revealed a significantly lower incidence rate in the DV cohort than in the matched control cohort (log-rank $p=0.035$; Fig. 3a). Furthermore, the Kaplan-Meier estimates of pSS-free survival revealed a significantly lower incidence rate in the DV cohort than in the matched control cohort (log-rank $p=0.0049$; Fig. 3e). The Kaplan-Meier estimates of other systemic disease-free survival revealed a nonsignificantly lower incidence rate in the DV cohort than in the matched control cohort (Fig $3 \mathrm{~b}, \mathrm{c}, \mathrm{d}$, and f).

\section{Discussion}

According to our review of the relevant literature, this is the first nationwide population-based study to evaluate the relationship between DV infection and the risk of ADs. The results of this study demonstrated that patients with DV infection had a lower risk of pSS than those without DV infection. The DV cohort had an approximately $70 \%$ lower risk of pSS than the control group, with an adjusted HR of 0.30 after adjusting for age, sex, and comorbidities. On the basis of this result, we contended that DV infection has a protective effect that reduces the risk of pSS.

DV infection can cause abnormal immune responses. Autoimmunity is characterized by autoantibody production and the activation of autoreactive lymphocytes. Other studies have indicated that the onset of the autoimmune response in dengue is a part of the pathogenesis of the disease that can affect various organs and systems [6,7]. These findings are formulated in a hypothesis concerning the possible role of DVs in the induction and maintenance of autoimmunity in ADs. However, our study determined that DV infection decreases the risk of ADs such as pSS.

Establishing a direct epidemiological association between microbial infections and autoimmune disorders is difficult. Attempts have largely been unsuccessful so far because of multiple predicaments. First, both patients suffering from ADs and healthy individuals undergo multiple infections during their lifetime. Most are cleared by the time of disease diagnosis. Thus, viral infections can be considered "hit and run" events that leave no precise evidence to establish the patient's history of prior infections. Second, genetic factors such as the MHC haplotype not only are directly responsible for disease susceptibility but also profoundly influence the antiviral immune response. A third factor that adds to the complexity is that infections are less likely to directly initiate autoimmunity but rather accelerate pre-existing autoimmune conditions that then progress to clinical diseases. This implies that multiple sequential events could be necessary to precipitate disease and further complicate attempts to establish firm proof for the involvement of environmental factors. Fourth, the precise timing, location, and magnitude of inflammation and viral strain might all play key roles. Indeed, some studies 
Table 1 Baseline characteristics of patients with dengue fever and age, sex, and residence matched to the comparison group

\begin{tabular}{|c|c|c|c|}
\hline & $\begin{array}{l}\text { Comparison group } \\
(n=117,460)\end{array}$ & $\begin{array}{l}\text { Dengue group } \\
(n=29,365)\end{array}$ & $p$ value \\
\hline \multicolumn{4}{|l|}{ Age } \\
\hline Mean, SD & $44.12,19.05$ & $44.13,19.05$ & 0.9984 \\
\hline \multicolumn{4}{|l|}{ Age group, $n(\%)$} \\
\hline $0-20$ & $15,450(13.15)$ & $3865(13.16)$ & 1.0000 \\
\hline $20-29$ & $14,817(12.61)$ & $3711(12.64)$ & \\
\hline $30-39$ & $17,731(15.10)$ & $4426(15.07)$ & \\
\hline $40-49$ & $19,759(16.82)$ & $4936(16.81)$ & \\
\hline $50-59$ & $22,977(19.56)$ & $5741(19.55)$ & \\
\hline $60-69$ & $17,339(14.76)$ & $4352(14.82)$ & \\
\hline $70-79$ & $7573(6.45)$ & $1881(6.41)$ & \\
\hline 80 & $1814(1.54)$ & $453(1.54)$ & \\
\hline Sex, $n(\%)$ & & & 0.9897 \\
\hline Male & $59,532(50.68)$ & $14,883(50.68)$ & \\
\hline Female & $57,928(49.32)$ & $14,482(49.32)$ & \\
\hline \multicolumn{4}{|l|}{ CCI score, $n(\%)$} \\
\hline Mean, SD & $0.62,1.29$ & $0.78,1.31$ & $<0.0001$ \\
\hline Min, $\max$ & $(0,15)$ & $(0,14)$ & \\
\hline 0 & $77,312(65.82)$ & $16,306(55.53)$ & $<0.0001$ \\
\hline 1 & $25,573(21.77)$ & $8221(28.00)$ & \\
\hline $2-3$ & $10,702(9.11)$ & $3803(12.95)$ & \\
\hline $4-6$ & $2833(2.41)$ & $803(2.73)$ & \\
\hline $7-10$ & $810(0.69)$ & $179(0.61)$ & \\
\hline 11 & $230(0.20)$ & $53(0.18)$ & \\
\hline Periodontitis & $65,118(55.44)$ & $18,562(63.21)$ & $<0.0001$ \\
\hline Residence, $n(\%)$ & & & 1.0000 \\
\hline Kaohsiung & $92,696(78.92)$ & $23,174(78.92)$ & \\
\hline Tainan & $13,176(11.22)$ & $3294(11.22)$ & \\
\hline Pingtung & $5364(4.57)$ & $1341(4.57)$ & \\
\hline New Taipei & $1324(1.13)$ & $331(1.13)$ & \\
\hline Taipei & $1272(1.08)$ & $318(1.08)$ & \\
\hline Taichung & $788(0.67)$ & $197(0.67)$ & \\
\hline Taoyuan & $728(0.62)$ & $182(0.62)$ & \\
\hline Penghu & $488(0.42)$ & $122(0.42)$ & \\
\hline Changhua & $344(0.29)$ & $86(0.29)$ & \\
\hline Yunlin & $176(0.15)$ & $44(0.15)$ & \\
\hline Hsinchu City & $172(0.15)$ & $43(0.15)$ & \\
\hline Nantou & $144(0.12)$ & $36(0.12)$ & \\
\hline Chiayi County & $144(0.12)$ & $36(0.12)$ & \\
\hline Miaoli & $136(0.12)$ & $34(0.12)$ & \\
\hline Taitung & $120(0.10)$ & $30(0.10)$ & \\
\hline Yilan & $92(0.08)$ & $23(0.08)$ & \\
\hline Keelung & $88(0.07)$ & $22(0.07)$ & \\
\hline Hsinchu City & $80(0.07)$ & $20(0.07)$ & \\
\hline Chiayi City & $64(0.05)$ & $16(0.05)$ & \\
\hline Hualien & $40(0.03)$ & $10(0.03)$ & \\
\hline Kinmen, Lienchiang & $24(0.02)$ & $6(0.02)$ & \\
\hline
\end{tabular}

CCI, Charlson Comorbidity Index

have demonstrated that the modification of these parameters can change a disease-enhancing viral infection to the one that prevents diabetes $[27,28]$. As a last factor, one must recognize that certain infections might protect an individual from an autoimmune response rather than enhance it $[29,30]$. Thus, the entire infection history of each patient might determine the overall immune status that results in an AD.

Many experimental systems support the hygiene hypothesis, which postulates that infections caused by viruses and inflammation protect rather than induce/accelerate ADs. For example, injection with coxsackievirus can not only enhance [31] but also prevent diseases in the non-obese diabetic (NOD) mouse [32]. Furthermore, IFN- $\gamma$ or TNF- $\alpha$ has protective effects in experimental autoimmune encephalomyelitis models or diabetes models when administered late in the disease process [33, 34]. The hygiene hypothesis [35] addresses the relationship between the reduction in the incidence of infectious diseases and the increase in the incidence of allergic diseases and ADs, and the apparent protective effects of infections against immune-mediated diseases have clear clinical implications. A major problem with these correlations is that the infections contributing to protection or susceptibility are ill defined. Moreover, certain infectious agents can trigger allergic diseases or ADs. Although our retrospective study supports the hygiene hypothesis, two lines of research are needed. One should focus on strengthening the epidemiologic evidence, especially through the use of prospective studies. Some allergic diseases and ADs are amenable to prospective epidemiologic investigations because they occur early in life (such as atopic dermatitis, asthma, and type 1 diabetes), thereby reducing the survey time. The second line of research should examine the reduced incidence of selected allergic diseases and ADs by innocuous immunostimulation.

Many animal models have been generated to study the mechanisms of the process that protects an individual from ADs. Mechanistically, several factors may play a role. First, inflammation caused by viruses, bacteria, and especially by parasites such as helminthes can shift the Th1-Th2 balance toward a more immunosuppressive state. In these situations, regulatory $\mathrm{T}$ cells might be induced or augmented. Indeed, some studies have discovered evidence for regulatory cells with specificity for pathogens in Leishmania majo [36], HSV [37], and Friend retrovirus (murine leukemia virus) infections [38]. Second, inflammation might cause a massive hyperactivation of autoaggressive lymphocytes, which may lead to activation-induced cell death and diminish the systemic load of aggressive $T$ cells. The concept that repeated encounters with strong antigenic stimuli lead to contraction of an immune response is well established in viral infections where the primary response undergoes a major contraction after Ag has been eliminated [39, 40]. These considerations also imply that to enhance autoimmunity, just the right type of stimulus is required. Pushing aggressive $\mathrm{T}$ cells too much will result in their rapid death by apoptosis [29], whereas low-level stimuli such as those provided by molecular mimicry might expand dangerous $\mathrm{T}$ cell populations by circumventing excessive apoptosis [29]. Third, infection at another location might keep autoaggressive cells from reaching the site of autoimmune destruction. This last possibility may be responsible for 
Table 2 Outcome incidence in patients with DV and comparison group and results of the Cox model regression

\begin{tabular}{|c|c|c|c|c|c|c|}
\hline \multirow[b]{2}{*}{ Outcome } & \multicolumn{2}{|c|}{ Comparison group } & \multicolumn{2}{|c|}{ Dengue group } & \multirow[b]{2}{*}{ IRR } & \multirow[b]{2}{*}{ Adjusted HR $(95 \% \mathrm{CI})^{\mathrm{b}}$} \\
\hline & $n(\%)$ & Incidence $^{\mathrm{a}}$ & $n(\%)$ & Incidence $^{\mathrm{a}}$ & & \\
\hline Systemic autoimmune diseases & $813(0.69)$ & 15.03 & $172(0.59)$ & 12.60 & 0.84 & $0.81(0.69-0.96)^{*}$ \\
\hline Ankylosing spondylitis & $383(0.33)$ & 7.05 & $89(0.30)$ & 6.50 & 0.92 & $0.89(0.70-1.13)$ \\
\hline Psoriasis & $237(0.20)$ & 4.36 & $52(0.18)$ & 3.79 & 0.87 & $0.84(0.62-1.15)$ \\
\hline Inflammatory myopathy & $-(0.00)$ & - & $0(0.00)$ & 0.00 & & NA \\
\hline Rheumatoid arthritis & $91(0.08)$ & 1.67 & $14(0.05)$ & 1.02 & 0.61 & $0.60(0.34-1.08)$ \\
\hline Sjogren's syndrome & $80(0.07)$ & 1.47 & $7(0.02)$ & 0.51 & 0.35 & $0.30(0.13-0.67)^{* *} \mathrm{a}++$ \\
\hline Systemic lupus erythematosus & $29(0.02)$ & 0.53 & $11(0.04)$ & 0.80 & 1.51 & $1.65(0.74-3.71)$ \\
\hline Systemic sclerosis & $11(0.01)$ & - & $-(0.00)$ & - & & NA \\
\hline Systemic vasculitis & $-(0.00)$ & - & $-(0.01)$ & - & & NA \\
\hline Organ-specific autoimmune diseases & $2212(1.88)$ & 41.35 & $597(2.03)$ & 44.37 & 1.07 & $1.03(0.94-1.13)$ \\
\hline Addison's disease & $319(0.27)$ & 5.87 & $76(0.26)$ & 5.55 & 0.95 & $0.96(0.74-1.25)$ \\
\hline Autoimmune hemolytic anemia & $15(0.01)$ & 0.28 & $5(0.02)$ & 0.36 & 1.32 & $1.07(0.33-3.47)$ \\
\hline Diabetes mellitus type 1 & $894(0.76)$ & 16.52 & $236(0.80)$ & 17.32 & 1.05 & $1.00(0.86-1.16)$ \\
\hline Graves' disease & $275(0.23)$ & 5.06 & $80(0.27)$ & 5.84 & 1.15 & $1.12(0.87-1.45)$ \\
\hline Hashimoto's thyroiditis & $430(0.37)$ & 7.92 & $106(0.36)$ & 7.75 & 0.98 & $0.90(0.72-1.12)$ \\
\hline Henoch-Schonlein purpura & $13(0.01)$ & 0.24 & $8(0.03)$ & 0.58 & 2.44 & $2.20(0.83-5.81)$ \\
\hline Immune thrombocytopenic purpura & $52(0.04)$ & 0.95 & $22(0.07)$ & 1.60 & 1.68 & $1.59(0.94-2.69)$ \\
\hline Autoimmune hepatitis & $293(0.25)$ & 5.39 & $92(0.31)$ & 6.73 & 1.25 & $1.22(0.95-1.56)$ \\
\hline Myasthenia gravis & $14(0.01)$ & 0.26 & $3(0.01)$ & 0.22 & 0.85 & $0.85(0.23-3.14)$ \\
\hline Inflammatory bowel disease & $13(0.01)$ & 15.03 & $0(0.00)$ & 12.60 & 0.84 & NA \\
\hline
\end{tabular}

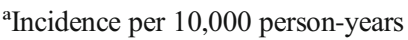

${ }^{\mathrm{b}}$ Matched age, gender, residence and adjusted by CCI score, and periodontitis (yes/no)

*For HR, raw $p=0.03$

**For HR, raw $P<0.001$. a: Hochberg corrected $p<0.001^{++}$

$C I$, confidence interval; $H R$, hazard ratio; IRR, incidence rate ratio; $N A$, not applicable; inflammatory bowel disease (Crohn's disease or regional enteritisunspecified, ulcerative colitis), systemic vasculitis (Behçet's disease, polyarteritis nodosa, Takayasu arteritis, or temporal arteritis)

the abrogation of T1D in NOD mice after lymphocytic choriomeningitis virus infection, as was initially observed more than a decade ago [41, 42]. Taking all this present evidence together, such animal models form a platform from which possible therapeutic treatments that target the termination or control of the protective process can be evaluated. They can serve as useful tools to understand the mechanisms that could underlie complex human autoimmune disorders and are well suited to establish proof of principle. The aforementioned underlying mechanisms are multiple and complex. They include decreased consumption of homeostatic factors and immunoregulation, involving various regulatory $\mathrm{T}$ cell subsets and toll-like receptor stimulation [35]. These mechanisms could originate, to some extent, from changes in microbiota caused by changes in lifestyle, particularly in IBD. Taken together, these data open new therapeutic perspectives for the prevention of ADs.
The pSS is an AD characterized by the activation of minor salivary gland (MSG) epithelial cells and B and T lymphocytic infiltrates. These findings have long encouraged the hypothesis that persistent viral infection of the MSG epithelial cells may drive the autoimmune response; however, the identity of that virus has remained elusive. Over the past decades, an extensive debate has developed concerning the possible role of various viral strains in the induction or maintenance of several ADs, including pSS [43, 44]. Infections caused by coxsackievirus strains B3 and B4 play a role in the initiation of SS [45]. Furthermore, several lines of epidemiological, serological, and experimental evidence implicate retroviral infections, especially HTLV-1, HIVs, HIAP-I, and HRV-5, as triggering factors for the development of SS [46]. However, our study does not support these findings. The relationship between infections and autoimmunity is complex. Current evidence indicates that microbes can initiate, enhance, or conversely, abrogate autoimmunity [47]. 
Fig. 2 Kaplan-Meier plots of cumulative incidence of organspecific ADs. a Organ-specific ADs. b Addison's disease. c Autoimmune hemolytic anemia. d Diabetes mellitus type 1. e Graves' disease. f Hashimoto's thyroiditis. g Autoimmune hepatitis. h Myasthenia gravis (a) Organ-specific ADs

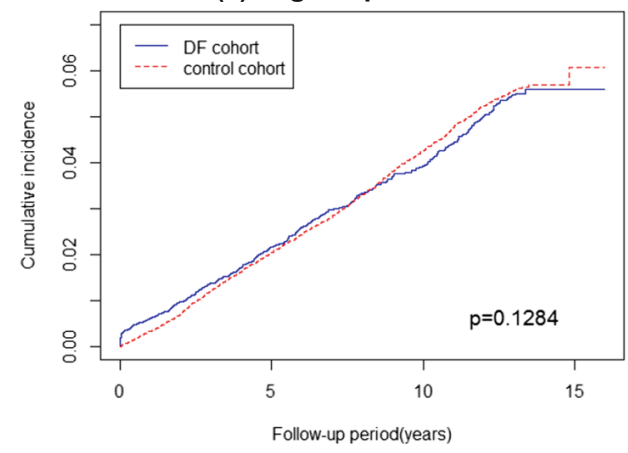

(c) Autoimmune hemolytic anemia

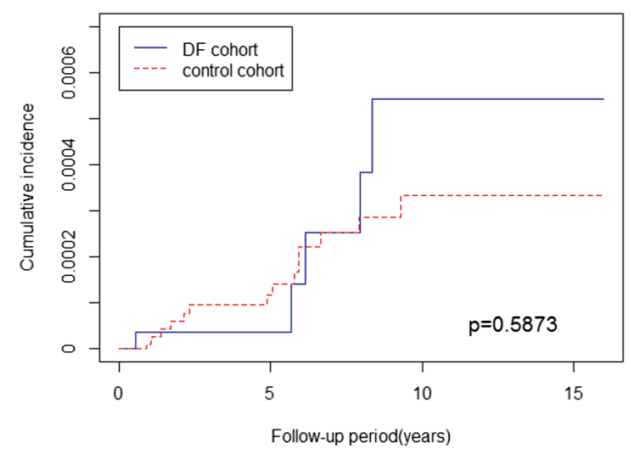

(e) Graves' disease

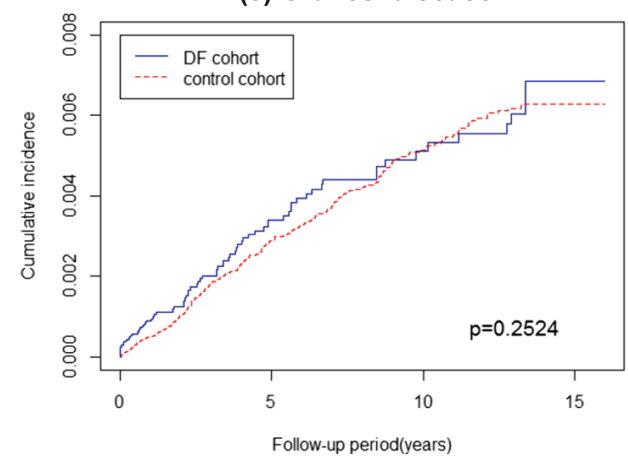

(g) Autoimmune hepatitis

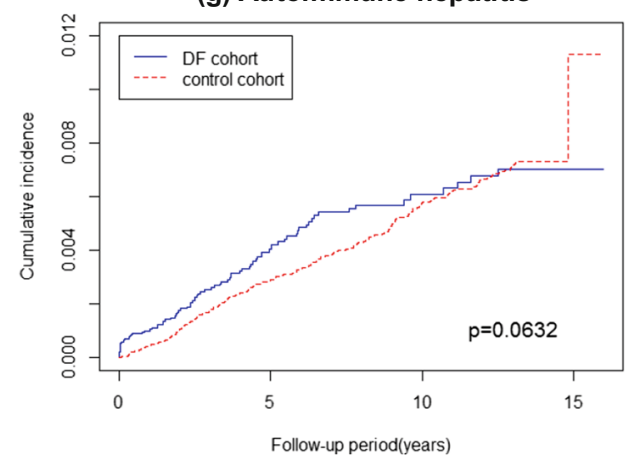

(b) Addison's disease

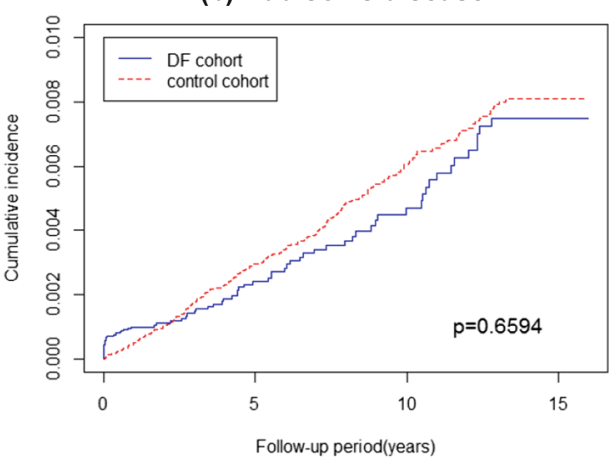

(d) Diabetes mellitus type 1

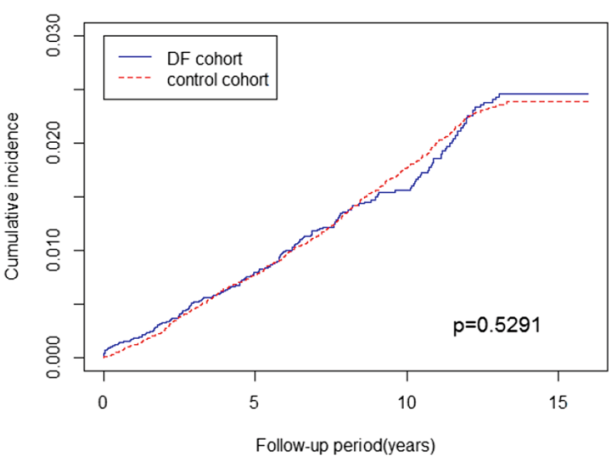

(f) Hashimoto's thyroiditis

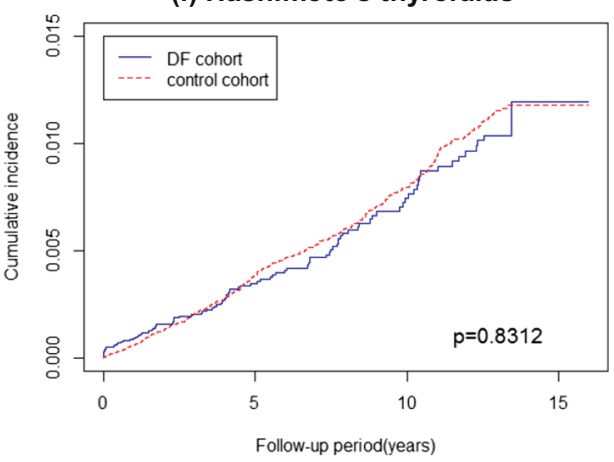

(h) Myasthenia gravis

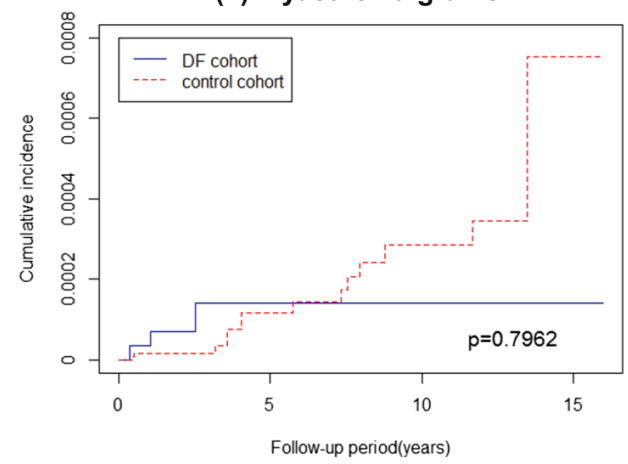

The strengths of the present study include large sample size, a large validation cohort, and the long-term ascertainment of concurrent ADs. However, the present study has some limitations. First, although the Bureau of NHI routinely and randomly monitors patient charts to ensure the quality of claims from all medical institutions, the possibility of miscoding or misclassification cannot be completely ruled out. However, such bias would apply to both the DV and 
Fig. 3 Kaplan-Meier plots of cumulative incidence of systemic ADs. a Systemic ADs. b Ankylosing spondylitis. c Psoriasis. d Rheumatoid arthritis. e Sjogren's syndrome. f Systemic lupus erythematosus (a) Systemic ADs

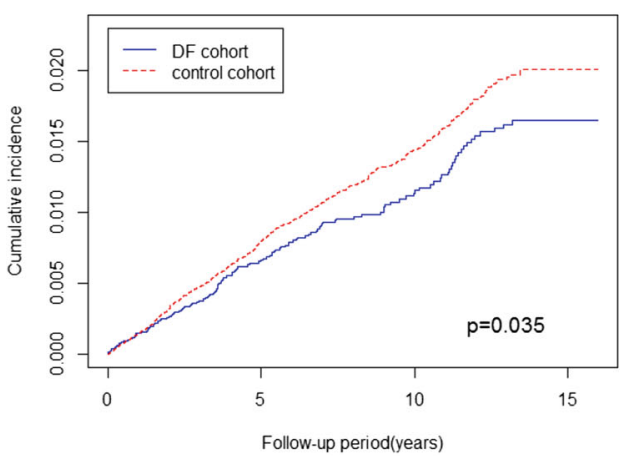

(c) Psoriasis

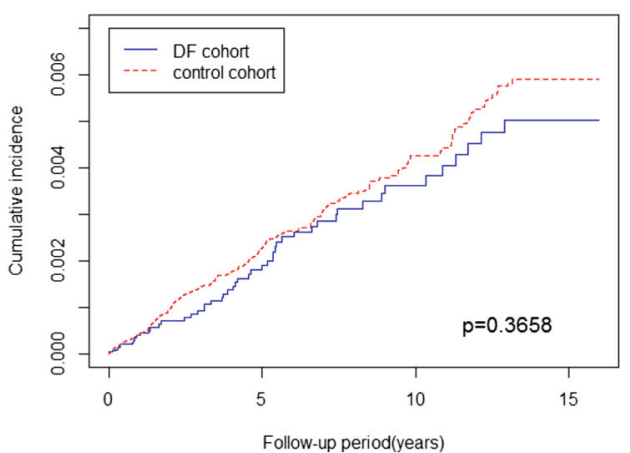

(e) Sjögren's syndrome

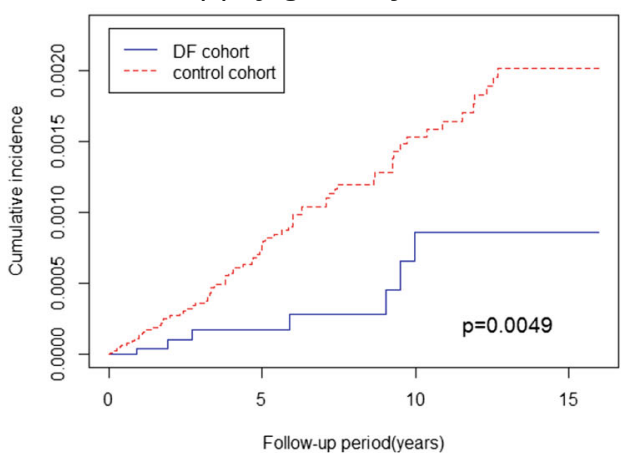

(b) Ankylosing spondylitis

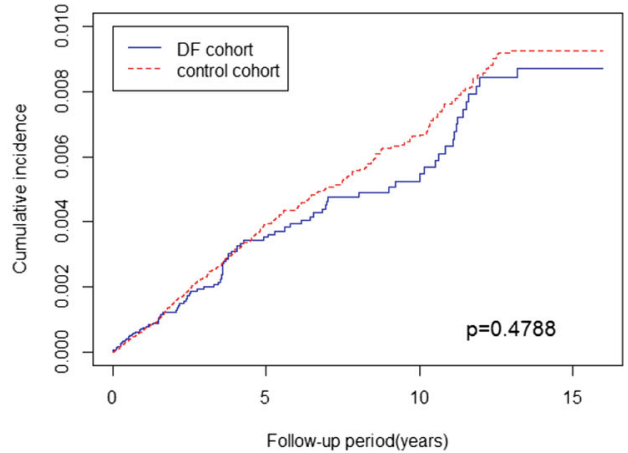

(d) Rheumatoid arthritis

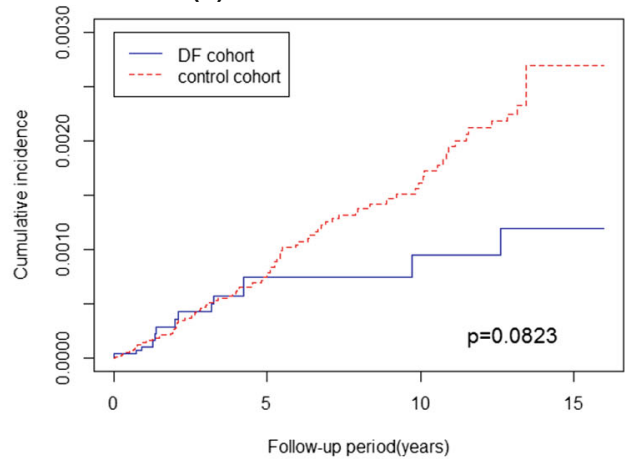

(f) Systemic lupus erythematosus

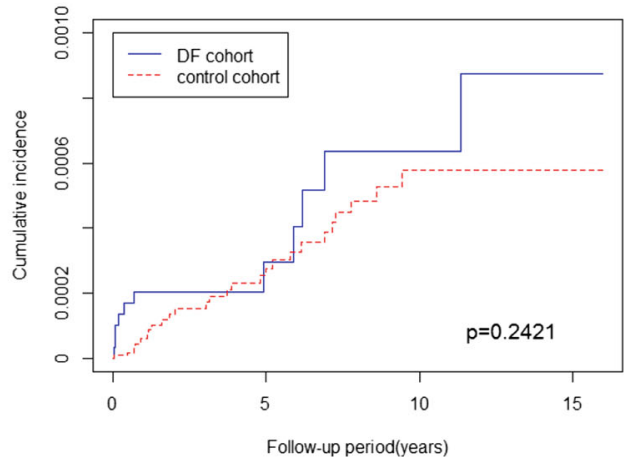

control cohorts, and therefore, the present findings are expected to underestimate rather than overestimate the magnitude of the association between DVs and ADs. Second, the relationship between the severity of DVs and ADs could not be analyzed. Additional prospective studies are warranted to confirm whether the severity of DVs increases the risk of ADs. Finally, some important information regarding laboratory or clinical data was not readily available in the administrative database, such as DV infection subtype data. Therefore, the relationship between the various subtypes of DV infection (dengue fever or dengue hemorrhagic fever) and ADs remains unclear. Additional studies are warranted to explore this association.

In conclusion, this nationwide long-term cohort study revealed an association between DV infection and a lower risk of $\mathrm{pSS}$. The protective immune mechanisms of DVs require elucidation. These findings may lead to the development of novel therapeutic strategies for ADs.

Author's contributions Chi-Ching Chang contributed to study conception and design, article drafting, critical article revision for substantive content, and the final approval of the submitted version. Yu-Chun Yen contributed to data interpretation, critical article revision for substantive content, and the final approval of the submitted version. Cheng-Yi Lee and Chiou-Feng Lin contributed to data analysis, critical article revision for substantive content, and the final approval of the submitted version. Chao-Ching Huang contributed to data analysis, article drafting, and the final approval of the submitted version. Ching-Wen Tsai contributed to data analysis. Ting-Wu Chuang and Chyi-Huey Bai were responsible for study conception and design, complete data analysis, critical article revision for substantive content, and correspondence for the final approval of the submitted version. 


\section{Compliance with ethical standards}

Disclosures None.

Open Access This article is licensed under a Creative Commons Attribution 4.0 International License, which permits use, sharing, adaptation, distribution and reproduction in any medium or format, as long as you give appropriate credit to the original author(s) and the source, provide a link to the Creative Commons licence, and indicate if changes were made. The images or other third party material in this article are included in the article's Creative Commons licence, unless indicated otherwise in a credit line to the material. If material is not included in the article's Creative Commons licence and your intended use is not permitted by statutory regulation or exceeds the permitted use, you will need to obtain permission directly from the copyright holder. To view a copy of this licence, visit http://creativecommons.org/licenses/by/4.0/.

\section{References}

1. Simmons CP, Farrar JJ, van Vinh Chau N, Wills B (2012) Dengue. N Engl J Med 366:1423-1432

2. Dengue: Guidelines for diagnosis, treatment, prevention and control. New edition. World Health Organization; 2009

3. Ranjit S, Kissoon N (2011) Dengue hemorrhagic fever and shock syndromes. Pediatr Crit Care Med 12:90-100

4. García G, González N, Pérez AB, Sierra B, Aguirre E, Rizo D et al (2011) Long-term persistence of clinical symptoms in dengueinfected persons and its association with immunological disorders. Int J Infect Dis 15:38-43

5. Wan SW, Lin CF, Yeh TM, Liu CC, Liu HS, Wang S, Ling P, Anderson R, Lei HY, Lin YS (2013) Autoimmunity in dengue pathogenesis. J Formos Med Assoc 112(1):3-11

6. Lin CF, Wan SW, Cheng HJ, Lei HY, Lin YS (2006) Autoimmune pathogenesis in dengue virus infection. Viral Immunol 19(2):127132

7. Liu IJ, Chiu CY, Chen YC, Wu HC (2011) Molecular mimicry of human endothelial cell antigen by autoantibodies to nonstructural protein 1 of dengue virus. J Biol Chem 286:9726e36

8. Kivity S, Arango MT, Ehrenfeld M, Tehori O, Shoenfeld Y, Anaya JM, Agmon-Levin N (2014) Infection and autoimmunity in Sjogren's syndrome: a clinical study and comprehensive review. J Autoimmun 51:17-22

9. Versini M, Jeandel PY, Bashi T, Bizzaro G, Blank M, Shoenfeld Y (2015) Unraveling the hygiene hypothesis of helminthes and autoimmunity: origins, pathophysiology, and clinical applications. BMC Med 13:81

10. Martina BE, Koraka P, Osterhaus AD (2009) Dengue virus pathogenesis: an integrated view. Clin Microbiol Rev 22(4):564-581

11. Cheng TM (2009) Taiwan's National Health Insurance system: high value for the dollar. In: Okma KGH, Crivelli L (eds) Six countries, six reform models: the health reform experience of Israel, the Netherlands, New Zealand, Singapore, Switzerland and Taiwan. World Scientific, Hackensack, pp 71-204

12. Chang CC, Chang YS, Chen WS, Chen YH, Chen JH (2016) Effects of annual influenza vaccination on morbidity and mortality in patients with systemic lupus erythematosus: a nationwide cohort study. Sci Rep 6:37817

13. Chang CC, Chiou CS, Lin HL, Wang LH, Chang YS, Lin HC (2015) Increased risk of acute pancreatitis in patients with rheumatoid arthritis: a population-based cohort study. PLoS One 10(8): e0135187
14. Fridkis-Hareli M (2008) Immunogenetic mechanisms for the coexistence of organ-specific and systemic autoimmune diseases. J Autoimmune Dis 5:1

15. Hochberg MC (1997) Updating the American College of Rheumatology revised criteria for the classification of systemic lupus erythematosus. Arthritis Rheum 40:1725

16. Arnett FC, Edworthy SM, Bloch DA, Mcshane DJ, Fries JF, Cooper NS, Healey LA, Kaplan SR, Liang MH, Luthra HS, Medsger TA, Mitchell DM, Neustadt DH, Pinals RS, Schaller JG, Sharp JT, Wilder RL, Hunder GG (1988) The American Rheumatism Association 1987 revised criteria for the classification of rheumatoid arthritis. Arthritis Rheum 31:315-324

17. American Rheumatism Association Diagnostic and Therapeutic Criteria Committee (1980) Preliminary criteria for the classification of systemic sclerosis (scleroderma). Subcommittee for scleroderma criteria of the American Rheumatism Association Diagnostic and Therapeutic Criteria Committee. Arthritis Rheum 23:581-590

18. Vitali C, Bombardieri S, Jonsson R, Moutsopoulos HM, Alexander EL, Carsons SE, Daniels TE, Fox PC, Fox RI, Kassan SS, Pillemer SR, Talal N, Weisman MH, European Study Group on Classification Criteria for Sjögren's Syndrome (2002) Classification criteria for Sjogren's syndrome: a revised version of the European criteria proposed by the American-European consensus group. Ann Rheum Dis 61:554-558

19. Bohan A, Peter JB (1975) Polymyositis and dermatomyositis (second of two parts). N Engl J Med 292:403-407

20. Bohan A, Peter JB (1975) Polymyositis and dermatomyositis (first of two parts). N Engl J Med 292:344-347

21. International Study Group for Behçet's Disease (1990) Criteria for diagnosis of Behçet's disease. Lancet. 335:1078-1080

22. Hunder GG, Bloch DA, Michel BA, Stevens MB, Arend WP, Calabrese LH, Edworthy SM, Fauci AS, Leavitt RY, Lie JT (1990) The American College of Rheumatology 1990 criteria for the classification of giant cell arteritis. Arthritis Rheum 33:11221128

23. Leavitt RY, Fauci AS, Bloch DA, Michel BA, Hunder GG, Arend WP, Calabrese LH, Fries JF, Lie JT, Lightfoot RW Jr (1990) The American College of Rheumatology 1990 criteria for the classification of Wegener's granulomatosis. Arthritis Rheum 33:11011107

24. Arend WP, Michel BA, Bloch DA, Hunder GG, Calabrese LH, Edworthy SM, Fauci AS, Leavitt RY, Lie JT, Lightfoot RW Jr (1990) The American College of Rheumatology 1990 criteria for the classification of Takayasu arteritis. Arthritis Rheum 33:1129 1134

25. Kuo CF, Grainge MJ, Valdes AM, See LC, Luo SF, Yu KH, Zhang W, Doherty M (2015) Familial aggregation of systemic lupus erythematosus and coaggregation of autoimmune diseases in affected families. JAMA Intern Med 175:1518-1526

26. Kuo CF, Grainge MJ, Valdes AM, See LC, Luo SF, Yu KH, Zhang W, Doherty M (2015) Familial risk of Sjögren's syndrome and coaggregation of autoimmune diseases in affected families: a nationwide population study. Arthritis Rheumatol 67:1904-1912

27. Tracy S, Drescher KM, Chapman NM, Kim KS, Carson SD, Pirruccello S, Lane PH, Romero JR, Leser JS (2002) Toward testing the hypothesis that group B coxsackieviruses (CVB) trigger insulin-dependent diabetes: inoculating nonobese diabetic mice with CVB markedly lowers diabetes incidence. J Virol 76:1209712111

28. Horwitz MS, Bradley LM, Harbertson J, Krahl T, Lee J, Sarvetnick N (1998) Diabetes induced by coxsackie virus: initiation by bystander damage and not molecular mimicry. Nat Med 4:781-785

29. Christen U, Benke D, Wolfe T, Rodrigo E, Rhode A, Hughes AC, Oldstone MB, Von Herrath MG (2004) Cure of prediabetic mice by viral infections involves lymphocyte recruitment along an IP-10 gradient. J Clin Invest 113:74-84 
30. Zaccone P, Fehervari Z, Jones FM, Sidobre S, Kronenberg M, Dunne DW, Cooke A (2003) Schistosoma mansoni antigens modulate the activity of the innate immune response and prevent onset of type 1 diabetes. Eur J Immunol 33:1439-1449

31. Horwitz MS, La Cava A, Fine C, Rodriguez E, Ilic A, Sarvetnick N (2000) Pancreatic expression of interferon-_ protects mice from lethal coxsackievirus B3 infection and subsequent myocarditis. Nat Med 6:693-697

32. Tracy S, Drescher KM, Chapman NM, Kim KS, Carson SD, Pirruccello S, Lane PH, Romero JR, Leser JS (2002) Toward testing the hypothesis that group B coxsackie viruses (CVB) trigger insulin-dependent diabetes: inoculating nonobese diabetic mice with CVB markedly lowers diabetes incidence. J Virol 76:1209712111

33. Jacob CO, Aiso S, Michie SA, McDevitt HO, Acha-Orbea H (1990) Prevention of diabetes in nonobese diabetic mice by tumor necrosis factor (TNF): similarities between TNF- $\alpha$ and interleukin 1. Proc Natl Acad Sci U S A 87:968-972

34. Christen U, Wolfe T, Mohrle U, Hughes AC, Rodrigo E, Green EA, Flavell RA, von Herrath MG (2001) A dual role for TNF- $\alpha$ in type 1 diabetes: islet-specific expression abrogates the ongoing autoimmune process when induced late but not early during pathogenesis. J Immunol 166:7023-7032

35. Okada H, Kuhn C, Feillet H, Bach JF (2010) The 'hygiene hypothesis' for autoimmune and allergic diseases: an update. Clin Exp Immunol 160(1):1-9

36. Mendez S, Reckling SK, Piccirillo CA, Sacks D, Belkaid Y (2004) Role for CD4_CD25_regulatory $\mathrm{T}$ cells in reactivation of persistent leishmaniasis and control of concomitant immunity. J Exp Med 200:201-210

37. Toka FN, Suvas S, Rouse BT (2004) CD4 CD25 T cells regulate vaccinegenerated primary and memory $\overline{\mathrm{CD}} 8 \overline{\mathrm{T}}$-cell responses against herpes simplex virus type 1. J Virol 78:13082-13089
38. Dittmer U, He H, Messer RJ, Schimmer S, Olbrich AR, Ohlen C, Greenberg PD, Stromnes IM, Iwashiro M, Sakaguchi S et al (2004) Functional impairment of CD8 T cells by regulatory T cells during persistent retroviral infection. Immunity 20:293-303

39. Murali-Krishna K, Altman JD, Suresh M, Sourdive DJ, Zajac AJ, Miller JD, Slansky J, Ahmed R (1998) Counting antigen-specific CD8 T cells: a reevaluation of bystander activation during viral infection. Immunity 8:177-187

40. Homann D, Teyton L, Oldstone MB (2001) Differential regulation of antiviral T-cell immunity results in stable CD8_ but declining CD4 T-cell memory. Nat Med 7:913-919

41. Oldstone MB (1990) Viruses as therapeutic agents. I Treatment of nonobese insulin- dependent diabetes mice with virus prevents insulin-dependent diabetes mellitus while maintaining general immune competence. J Exp Med 171:2077-2089

42. Oldstone MB (1988) Prevention of type I diabetes in nonobese diabetic mice by virus infection. Science 239:500-502

43. Manoussakis MN, Moutsopoulos HM (2001) Sjogren's syndrome: current concepts. Adv Intern Med 47:191-217

44. Zinkernagel RM (2002) Antiinfection immunity and autoimmunity. Ann N Y Acad Sci 958:3-6

45. Triantafyllopoulou A, Tapinos N, Moutsopoulos HM (2004) Evidence for coxsackievirus infection in primary Sjogren's syndrome. Arthritis Rheum 50:2897-2902

46. Sipsas NV, Gamaletsou MN, Moutsopoulos HM (2011) Is Sjögren's syndrome a retroviral disease? Arthritis Res Ther 13:212

47. Christen U, von Herrath MG (2005) Infections and autoimmunitygood or bad? J Immunol 174(12):7481-7486

Publisher's note Springer Nature remains neutral with regard to jurisdictional claims in published maps and institutional affiliations.

\section{Affiliations}

\section{Chi-Ching Chang ${ }^{1,2}$ (1) $\cdot$ Yu-Chun Yen ${ }^{3} \cdot$ Cheng-Yi Lee ${ }^{4,5} \cdot$ Chiou-Feng Lin $^{6,7} \cdot$ Chao-Ching Huang ${ }^{8} \cdot$ Ching Wen Tsai $^{3}$. Ting-Wu Chuang ${ }^{9} \cdot$ Chyi-Huey Bai ${ }^{10}$}

1 Division of Allergy, Immunology and Rheumatology, Department of Internal Medicine, School of Medicine, College of Medicine, Taipei Medical University, Taipei, Taiwan

2 Division of Rheumatology, Immunology and Allergy, Department of Internal Medicine, Taipei Medical University Hospital, Taipei, Taiwan

3 Research Center of Biostatistics, College of Management, Taipei Medical University, Taipei, Taiwan

4 Epidemic Intelligence Center, Taiwan Centers for Disease Control, Ministry of Health and Welfare, Taipei, Taiwan

5 Institute of Health Policy and Management, College of Public Health, National Taiwan University, Taipei, Taiwan
6 Department of Microbiology and Immunology, School of Medicine, College of Medicine, Taipei Medical University, Taipei, Taiwan

7 Graduate Institute of Medical Sciences, College of Medicine, Taipei Medical University, Taipei, Taiwan

8 Department of Pediatrics, School of medicine, College of Medicine, Taipei Medical University, Taipei, Taiwan

9 Department of Molecular Parasitology and Tropical Diseases, School of Medicine, College of Medicine, Taipei Medical University, Taipei, Taiwan

10 Department of Public Health, School of Public Health, College of Public Health, Taipei Medical University, 252, Wu-Hsing Street, Taipei, Taiwan 